Journal Article

\title{
Numerical simulation of non-Newtonian polymer film flow on a rotating spoked annulus
}

Miah, M. S., Hossain, M.S., Ashraf, M. A., Al-Assaf, S. and McMillan, A.J.

This article is published by Wiley. The definitive version of this article is available at: http://onlinelibrary.wiley.com/doi/10.1002/app.44943/full

This is the authors' submitted version.

\section{Recommended citation:}

Miah, M. S., Hossain, M.S., Ashraf, M. A., Al-Assaf, S. and McMillan, A.J. (2016), 'Numerical simulation of non-Newtonian polymer film flow on a rotating spoked annulus', Journal of Applied Polymer Science, published online 03 March 2017. DOI: 10.1002/app.44943 


\title{
Numerical simulation of non-Newtonian polymer film flow on a rotating spoked annulus
}

\author{
Md Salim Miah a, b, *, Mohammad Sayeed Hossain b, Muhammad Arif Ashraf b, Saphwan Al-Assaf a, c, \\ Alison McMillan a \\ a Applied Sciences, Computing and Engineering Division, Wrexham Glyndwr University, Wrexham, LL11 \\ 2AW, United Kingdom \\ ${ }^{\mathrm{b}}$ Aeronautical Engineering Department, Military Technological College, P.O. Box 262, P.C. 111, Sultanate \\ of Oman \\ ${ }^{c}$ Hydrocolloids Research Centre, Institute of food Science and Innovation, Faculty of Science \& \\ Engineering, University of Chester, Chester, $\mathrm{CH} 14 \mathrm{BJ}$, United Kingdom \\ * Corresponding Author's E-mail Address: MD.Salim@mtc.edu.om
}

\begin{abstract}
The current study presents results from a series of numerical analysis of non-Newtonian liquid film formation on a rotating spoked annulus. The film flow of a common type of polyester, Polyethylene Terephthalate (PET), on a vertically rotating disk was modelled numerically. Two different molecular weights, corresponding to different viscosities for PET, and under flow at different rotating speeds, were considered. The film thickness profile was obtained at different radial and angular positions of the rotating disk in the simulations, which is beneficial in calculating the volume of polymer taken up by the rotating disk and also in calculating the volumetric flow rates on the disk. Two types of disks including a standard solid disk and a number of hollow disk designs - spoked annuli were considered and the film flow modelled using the volume of fluid (VOF) computational fluid dynamics (CFD) analysis. The analyses of flow over spoked annulus designs highlighted the advantage of such designs over the use of a conventional solid disk. It was found that the variation in the film thickness for the spoked annulus was lower than that for the conventional solid disk. The parametric study also provided a favorable spoked annulus design for which the film thickness was essentially constant. A constant film thickness would provide a constant film flow which can be a benefit to many industrial applications.
\end{abstract}

\section{Keywords:}

Computational fluid dynamics (CFD) 
Non-Newtonian liquid

Polyethylene Terephthalate (PET)

Rotating spoked annulus

\section{Introduction}

Polyethylene Terephthalate (PET) is the most common type of polyester; its properties make it suitable for a wide array of product applications, particularly for the manufacture of plastic bottles and product packaging. The global PET market was estimated to be worth USD 23.3 billion in 2010 and is expected to reach USD 48.4 billion in 2016 [1, 2]. During the synthesis of the PET, a by-product component (Ethylene Glycol) and several other volatile species are produced [1]. In the last stage of the Polycondensation process, the reactor must remove this by-product in order to get a high quality polymer without impurities. To separate this component effectively from the melt polymer, the last stage reactor comprises a large horizontal cylindrical vessel, which contains several vertical disks mounted along a rotating shaft. Each disk facilitates the removal of volatile products, from the polymer by creating a film fluid over its surface where the diffusion of by- product takes place.

The flow of non-Newtonian liquids has gained considerable importance as a result of its application in different scientific and engineering industries including chemical industries, materials processing, coating industries and the food processing industries [3-6]. The flow behavior of the non-Newtonian liquids differs from those of the Newtonian liquids. The viscosity in non-Newtonian liquids is dependent on the shear rate for the applied shear stress [7]. In general, the polymers are highly viscous fluids and considered to follow non-Newtonian flow behavior. Depending on the structure and concentration, the conformation of a polymer solution might be considered like a bowl of spaghetti with the polymer chains entangled causing resistance to shearing of the fluid. A recent numerical study of Newtonian film flow on a vertically rotating solid disk was validated by an experimental study [8]. Numerical solutions for the non-Newtonian film flow are achieved using a similar CFD modelling approach [9-11].

To achieve an increased film thickness profile on the disk and an enhanced mass transfer, Woo and Yong [12] and Cai et al [13] proposed a new hollow rotating disk design. In the present study a number of hollow rotating disks (rotating spoked annulus) are modelled numerically. The numerical solutions are compared with the film flow on solid rotating disk and conclusions drawn on the influence on the volumetric film flow rate. Volume of fluid (VOF) modelling approach is applied employing commercially available CFD code Fluent [8]. It allows modelling of two immiscible fluids, air and PET in the present case. Numerical model is described in Section 2 with details of grid generation, boundary conditions and transient considerations. Numerical results of the non-Newtonian film flow on a standard solid disk are discussed in Section 3 together with a validation by previous experimental work. Section 4 provides a summary of the predicted 
film flow on a number of hollow disks and discusses their advantages over the use of a solid disk. Finally conclusions are drawn and summarized in Section 5.

\section{NOMENCLATURE}

$\begin{array}{lll}g & {\left[\mathrm{~m} / \mathrm{s}^{2}\right]} & \text { Gravitational acceleration } \\ h & {[\mathrm{~m}]} & \text { Thin film thickness } \\ h^{\prime} & {[-]} & \text { Dimensionless film thickness } \\ R & {[\mathrm{~m}]} & \text { Disk radius } \\ K & {\left[\text { Pa.s }^{n}\right]} & \text { Consistency index } \\ R^{\prime} & {[-]} & \text { Dimensionless disk radius } \\ n & {[-]} & \text { Power law index } \\ \dot{\gamma} & {\left[\mathrm{s}^{-1}\right]} & \text { Shear rate }\end{array}$

Special characters

\begin{tabular}{|c|c|c|}
\hline$\theta$ & {$\left[{ }^{0}\right]$} & Angular coordinate \\
\hline$\Omega$ & [rpm] & Rotating speed \\
\hline$\eta_{0}$ & [Pa.s] & Maximum viscosity \\
\hline$\eta_{\infty}$ & [Pa.s] & Minimum viscosity \\
\hline$X_{n}$ & {$[-]$} & Number average chain length \\
\hline$\sigma$ & {$[\mathrm{N} / \mathrm{m}]$} & Surface tension \\
\hline$\rho$ & {$\left[\mathrm{kg} / \mathrm{m}^{3}\right]$} & Density \\
\hline
\end{tabular}

Subscripts

$\begin{array}{ll}\text { CFD } & \text { Computational fluid dynamics } \\ \text { CFL } & \text { Courant-Friedrich-Lewy condition } \\ \text { VOF } & \text { Volume of fluid } \\ \text { PET } & \text { Polyethylene terephthalate }\end{array}$

\subsection{Materials}

The simulations were carried out for two polymer samples of different molecular weight (number-average chain length $X_{n}=69$ and $X_{n}=82$ ) corresponding to two different viscosities 47.3 and 86.6 Pa.s respectively [1]. 
To model the PET fluid, the non-Newtonian power law was used in order to set the parameters of the viscosity.

The power law equation is given by $[16,17]$ :

$$
\eta=K(\dot{\gamma})^{n-1}
$$

where, $\eta$ is non-Newtonian viscosity,

$K$ is consistency index,

$n$ is power law index and

$\dot{\gamma}$ is the shear rate.

The consistency index can be calculated by rearranging Eq. (1) to give:

$$
K=\eta(\dot{\gamma})^{(1-n)}
$$

For $X_{n}=69$,

$K=47.3 \times(10)^{(1-0.6)}=119$ Pa.s $^{n}$

For $X_{n}=82$,

$K=86.6 \times(10)^{(1-0.6)}=217.53$ Pa.s $^{n}$

The minimum viscosity limit $\eta_{\infty}$ is determined from Eq. (1)

$$
\eta_{\infty}=K(\dot{\gamma})^{n-1}
$$

For $X_{n}=69$,

$\eta_{\infty}=119 \times 10000^{(0.6-1)}=2.99 P a . . S$

For $X_{n}=82$,

$\eta_{\infty}=217.53 \times 10000^{(0.6-1)}=5.46$ Pa..s

A summary of the calculated values of the parameters used is provided in Table 1. 


\section{Numerical modelling}

A three dimensional model was developed to model the PET film flow over vertically rotating disk. The model was generated in GAMBIT 2.4.6 [8] and the simulations conducted in FLUENT 6.3 for two different polymers to analyze the effect of the molecular weight on the film formation. The simulations were run under different angular velocities (10 rpm and $20 \mathrm{rpm}$ ) to study their influence on film thickness.

A three dimensional design of a rotating disk inside the reactor of diameter $140 \mathrm{~mm}$ was constructed in this study, similar to the dimensions used in a previous experimental study [1]. As shown in Figure 1 the length of the reactor was $140 \mathrm{~mm}$. The diameter and the thickness of the disk were $127 \mathrm{~mm}$ and $4 \mathrm{~mm}$ respectively. The disk was partially immersed (34\% of the diameter of the disk) in the PET. The results of present numerical study were validated against previously published experimental result using the same method [1].

\subsection{Grid generation}

The quadrilateral elements were used to create the three dimensional mesh for the present model, since for a simple geometry, a high aspect ratio quadrilateral element is easily achievable and would perform more efficiently than with triangular-tetrahedral cells. To mesh the edges, both constant successive ratio and the first length were used. This allowed a very fine mesh near the disk surface and large cells at the outer border of the reactor. The film thickness measurement area is near the disk surface. A coarser mesh was used at the reactor borders to minimize the number of elements as shown in Figure 2.

In order to determine the necessary mesh size to provide an adequate size representation of the film thickness, the film thickness results obtained by Cheong and Choi [1] were used as a guideline. The article provided a minimum thickness value of $1 \mathrm{~mm}$, therefore, in the present model the minimum mesh size was maintained near the disk surface: see Figure 2(a), which gives a minimum value of $0.25 \mathrm{~mm}$, i.e. a quarter of the minimum thickness in order to generate a reasonably high quality mesh. This also ensured reliability in the results. The mesh size of the edges in other areas varied from $0.90 \mathrm{~mm}$ to $1.5 \mathrm{~mm}$, a similar range of the minimum thickness value. The total number of elements used in the CFD modelling for the solid disk analysis was 698,808 . Exhaustive grid independence studies were carried out and the current mesh was found to be sufficiently refined to capture the flow details [8].

The boundary conditions used for the current model are illustrated in Figure 3. Wall boundary conditions were used to bound fluid and solid regions. The fluid area was defined by interior boundary conditions. Symmetry boundary conditions were used for both sides of the reactor. The present study involves solving two phase flow problem. To do this, the volume of fluid (VOF) model was chosen. This model permits to model two immiscible fluids, air and PET in the present case. Each fluid phase is modeled as a volume 
fraction of each cell in the mesh. A single set of momentum equations is solved and the volume fraction of each of the fluids throughout the domain is obtained. These volume fractions were essential to predict the thickness of the film layer. An 'Implicit Scheme' was used. The disk was set as a 'moving wall' with a rotational absolute motion of $10 \mathrm{rpm}$ and $20 \mathrm{rpm}$. The contact angle used between the PET and the AIR was $90^{\circ}[14,15]$.

\subsection{Transient analysis considerations}

In order to ensure that the numerical simulation is stable, a careful selection of time step was required. For a minimum mesh size of $0.25 \mathrm{~mm}$, the Courant-Friedrichs-Lewy condition (CFL) [8] and the maximum angular velocity were used to calculate the time step. The time step size was taken at $10^{-3} \mathrm{~s}$, which satisfies the CFL condition and 40 iterations per time step were used.

\section{Vertically rotating solid disk}

In this section numerical results are provided for the non-Newtonian film flow on a vertically rotating solid disk. The simulation was carried out for the two different molecular weights of PET, $X_{n}=69$ and $X_{n}=82$ corresponding to two different viscosities 47.3 and $86.6 \mathrm{~Pa}$.s respectively. The thickness for each molecular weight and two different rotating speeds of $10 \mathrm{rpm}$ and $20 \mathrm{rpm}$ were tested.

The flow pattern of PET film can be noticed from the numerical simulation in Figure 4, where the disk is rotating in an anti-clockwise direction. The legend with a scale of 1 represents $100 \%$ PET while the scale 0 represents $100 \%$ air.

It is interesting to note that the center of the rotating pattern remains void of the film, which is almost circular area with a calculated radius of about $32 \%$ of the disk radius. The circular area in the center with no film, moves to the right side slightly when the rotation direction of the disk is anti-clockwise. This phenomenon of the film formation is similar to a typical film formation of a Newtonian fluid, however, the film thickness profile of the non-Newtonian liquid is expected to be different from that of the Newtonian liquid. Note that the PET film flow in the drag out and drag in region, unlike in the Newtonian film flow case, does not exhibit increased thickness and instability. .

\subsection{Validation using previous experimental study}

A brief validation of the current numerical solution is provided in the present section followed by a parametric study to investigate the influence of a number of parameters on the subsequent film flow thickness. 
The CFD modelling simulations are compared with the experimental results [1] in Figure 5. Note, the experimental data was extracted graphically.

The PET film pattern obtained from the CFD modelling simulations is reasonably consistent with those shown in published experimental results [1]. The average film thickness obtained by the CFD simulation is over-predicted by about $20 \%$ to $30 \%$, based on the results obtained for the angular positions of $60^{\circ}$ and $120^{\circ}$. The possible reason is thought to be due to numerical diffusion error. The film thickness at the angular positions of $60^{\circ}$ and $120^{\circ}$ is more stable and of more interest to the industrial applications and therefore comparisons to experiments are limited to these angles. For other angles, $0^{\circ}$ and $180^{\circ}$, the CFD simulation results show similar trend to the measurements but quantitatively they are different. This is due to the high instability of film flow in drag in and drag out regions [8].

3.2 Numerical results of thin film flow on a vertically rotating solid disk

Figures 7 to 10 provide a summary of the predicted PET film thickness variation for a number of different parameters including (1) angular position, (2) radial position, (3) rotating speed and (4) molecular weight corresponding to viscosity. Note, the film thickness profiles provided are non-dimensionalized and obtained by dividing the thickness by the disk radius and multiplied by a factor of 10 . The magnification was necessary to provide a meaningful number.

\subsubsection{Film thickness for different angular positions}

The PET film thickness profile for different angular positions is presented in Figure 6 . The film is thicker towards the edge of the disk for angular position $0^{\circ}$, and starts decreasing towards the center of the disk. With the increase of the angular position from $0^{\circ}$ to $180^{\circ}$, the film becomes thinner towards the edge of the disk and thicker towards the center of the disk. This is due to the gravity and drag out effect.

It is interesting to note that the film thickness for angular position at $60^{\circ}$ increases from the disk edge until $R^{\prime}=7$ and decreases again towards the center of the disk. This effect is not seen in a Newtonian film flow [8].

\subsubsection{Film thickness for different radial positions}

The PET film thickness for radial positions $R^{\prime}=7$ and $R^{\prime}=9$ decreases as the angular position increase from $0^{\circ}$ to $180^{\circ}$ as shown in Figure 7 . The reason for this kind of phenomenon of film formation is identified as the gravity effect, in case of the drag out, the film is thicker and for gravity the film starts decreasing with the increase in the angular position. 
It is interesting that for the radial position $R^{\prime}=5$, the film pattern is different. The film thickness increases with the increase in the angular position. The possible reason for this flow pattern may be due to the shear rate dependent viscosity of non-Newtonian liquid.

It is observed that film thickness variation at the radial position $\left(R^{\prime}=7\right)$ is more stable compared to the other radial positions, which indicates that a constant volume of polymer hold up by the disk can be obtained in this position. Estimation of polymer retention by the disk is useful in practical applications, e.g. in determining the position of the polymer collectors in the reactors during polymer processing $[18,19]$.

\subsubsection{Comparison of film thickness profile for different rotating speeds}

The PET film thickness increases with the increase in the rotating speeds of the disk as shown in Figure 8. This kind of flow pattern is similar to those for Newtonian film flow shown in [8]. The film pattern at the angular positions of $60^{\circ}$ and $120^{\circ}$ is similar with very low amount of film thickness variation under both the rotating speeds of the disk. The film formation at these two angular positions is said to be stable.

\subsubsection{Comparison of film thickness for different molecular weight}

Figure 9 shows the PET film thickness profile at two different molecular weights, $X_{n}=69$ and $X_{n}=82$. A limited variation of the film thickness profile with the change in the molecular weight of PET can be seen. The fluid with the higher molecular weight has a higher viscosity, and thus shows a thicker film profile than that of the lower molecular weight fluid. A slight variation is observed at $60^{\circ}$ angular position. The PET film thickness is higher for the lower molecular weight. The film thickness profile of the PET polymer is essentially similar in trend for both molecular weights.

\section{Vertically rotating spoked annulus designs}

It is desirable to increase the mass transfer of the film flow on the rotating spoked annulus, and also to obtain and ensure a stable film formation for an increased volumetric flow rate. In view of this, a rotating spoked annulus design was proposed by Cai, Wang and Zhu [13] and was further developed in the present study. The proposed rotating spoked annulus design is shown in Figure 10. Four hollow sections with four connectors were selected to provide sufficient support to the rotating disk when it is attached to a rotating shaft.

4.1 Design of vertically rotating hollow disks 
The shaft diameter was taken about $7 \%$ of the disk diameter $(9.5 \mathrm{~mm})$ according to the guideline from [1]. By adjusting the size and position of the hollow parts of the rotating disk, more stable and increased film thickness could be achieved. In the present study three different designs of hollow rotating disks of different radii were considered and their radius calculation is shown below, where $R$ is the disk annulus internal radius.

Hollow disk $1=0.5 \mathrm{R}=31.75 \mathrm{~mm}$

Hollow disk $2=0.6 \mathrm{R}=38.1 \mathrm{~mm}$

Hollow disk $3=0.7 \mathrm{R}=44.45 \mathrm{~mm}$

The schematic of the hollow rotating disk (hollow disk 1) with dimensions is provided in Figure 11. The dimension of the connector cross section was taken as $4 \times 4 \mathrm{~mm}^{2}$ to provide adequate support to the rotating disk.

A full three dimensional CFD model was constructed for the hollow rotating disk in the same way as with the solid rotating disk discussed in section 2.1. The dimensions of the disk and the reactor for both the hollow and solid disks were taken the same, however, based on the three different radii of the hollow parts, three designs of hollow rotating disks were consequently used. Thus, in terms of the level of hollowness, hollow disk 3 is more hollow than hollow disk 2, which in turn is more hollow than hollow disk 1.

For grid generation the same principle of the meshing of the solid disk was used. A boundary layer mesh was imposed with a minimum mesh size of $0.25 \mathrm{~mm}$ near the disk surface and the coarse mesh was maintained at the outer border of the reactor, Figures 12 and 13. In order to generate a structured grid with hexahedral cells the map mesh faces were used. The total number of elements used for hollow disk 1 , hollow disk 2 and hollow disk 3 were 719,569, 787,800 and 828,808 respectively. All the boundary conditions and the PET properties were considered same for the both solid and hollow rotating disks.

\subsection{Numerical result of thin film flow on vertically rotating hollow disks}

This section summarizes numerical results for the non-Newtonian film flow on a vertically rotating hollow disk. Comparison is made with the numerical result of the non-Newtonian film flow on the solid disk described in Section 3 in order to illustrate the influence of the different hollow disk designs.

Figure 14 shows the polymer PET melt flowing both around and through the vertically rotating hollow disk. As expected of the non-Newtonian fluid the film flow pattern for the hollow disk is also similar to the film flow pattern for the solid disk shown in Figure 4. The viscous force contributes to the drag out phase creating 
the thin film on the right section of the disk whereas the gravitational force contributes to the formation of a thick film at the drag in phase of the disk.

The influence of the degree of hollow disk design on the predicted film thickness is shown in Figure 15. The film thickness profiles for radial positions $R^{\prime}=7$ and $R^{\prime}=9$ are shown. Note, the rotational speed of $20 \mathrm{rpm}$ was used. Similar to the trend for the solid disk shown in Figure 7, the film thickness for the hollow disk also generally decreased with the angular position increasing from $0^{\circ}$ to $180^{\circ}$. Also shown in the figure is the variation of the film thickness with angular positions for the solid disk at $20 \mathrm{rpm}$ speed for comparison.

In general, the film thickness variations for the hollow disks were reduced compared to the solid disk. In particular, for the hollow disk 2 the film thickness did not vary significantly with the angular positions. The film thickness was essentially constant. This study illustrates the possibility of an optimum disk design which can benefit in a number of industries which rely on collection of liquids. It is interesting to note in Figure 15(a) that the film thickness variation between 1.2 and 2.6 radians for the hollow disk 3 and the solid disk were identical.

\section{Discussion}

A non-Newtonian power law, Eq. (1) similar to that used in $[16,17]$ was used to model the present PET fluid. The CFD simulation results were compared with the published experimental results [1] for the solid disk and shown in Figure 5. The predicted film thickness was about $20-30 \%$ higher than the measured. The difference was thought to be related to the numerical diffusion error [20]. The other reasons for this difference may include polymer molecules becoming aligned as they are stirred, this effect has not been considered in the numerical model. Furthermore thermal and memory effects in the polymer might also have contributed to the difference. More work needs to be done to fine tune the predicted results; however, the similar trend observed between the simulation and the experiment indicates that the solid disk analysis can reasonably be extended to consider different hollow disk designs.

The PET polymer with higher molecular weight has a higher viscosity and is likely to show a thicker film profile, as observed in Figure 9. With the exception of the $60^{\circ}$ angular position at the dimensionless radial position 7 the film thickness for the PET with higher molecular weight was higher. In general the average film thickness was relatively similar for both molecular weights. The possible reason for that is likely to be due to shear stress effect of the non-Newtonian fluid.

\section{Concluding remarks}


The PET film flow on a vertically rotating disk was modelled numerically. The numerical results were obtained for mixed PET fluids of two different molecular weights, and for different rotating speeds. The predicted film thickness profile was obtained at different radial and angular positions on the rotating annulus. From these predictions, the amount of polymer taken up onto the rotating annulus and also the volumetric flow rates onto the annulus can be calculated.

The present simulation results on the solid disk were validated by comparing the numerical results to an experimental study [1]. Although a difference of $20-30 \%$ was present a very similar trend existed between the numerical and the experimental results. That was encouraging to extend further the solid disk analysis and to consider the different hollow disk/spoked annulus designs. In general the predicted film thickness variations for the hollow disks were lower than that for the solid disk.

By considering three different annulus internal radii it was possible to carry out a limited optimization study. Three different disk designs revealed that the film thickness was essentially constant for the hollow disk 2 (variation less than $8 \%$ ).

The present study clearly highlighted the advantage of using hollow disk design over standard solid disk. A constant film thickness was achieved which could provide a uniform film flow and thereby benefit in industrial applications.

The present analysis can be further extended to investigate the effect of number of hollows on film formation and also to calculate the mass transfer efficiency in order to determine an optimum hollow disk design.

\section{Acknowledgments}

The authors acknowledge the time resource afforded by Military Technological College, Oman for preparation of this article. The authors also wish to acknowledge the contributions of Professor Xiaogang Yang to the project.

\section{References}

1. Seong III Cheong, Kyu Yong Choi., A study on the polymer layer forming phenomena in a rotating disk polycondensation reactor, Journal of Applied Polymer Science, 1995, 55, 1819-1826.

2. Transparency Market Research, 'Global Polyethylene Terephthalate (PET) Value Chain Market Analysis', 2012.

3. Chhabra, R. P. \& Richardson, J. F., Non-Newtonian flow in the process industries: Fundamentals and engineering applications, 1999. 
4. Smit, G. J. F., Plessis, J. P. D. \& Wilms, J. M., On the modelling of non-Newtonian purely viscous flow through high porosity synthetic foams, Chem. Eng. Science, 60, 2815-2819, 2005.

5. Sun D. W., Computational Fluid Dynamics in Food Processing, CRC Press, 2007.

6. Nemati, H., Ghanbarpour, M., Hajibabayi, M. \& Hemmatnezhad, Thin film flow of non-Newtonian fluids on a vertical moving belt using Homotopy Analysis Method, J. Eng. Science and Tech. Review, 2(1), 118-122, 2009.

7. Chhabra, R. P., Non-Newtonian Fluids: An Introduction, Department of Chemical Engineering, Indian Institute of Technology Kanpur, 2010.

8. MD Salim Miah, Saphwan Al-Assaf, Xiaogang Yang, Alison McMillan, Thin film flow on a vertically rotating disk of finite thickness partially immersed in a highly viscous liquid, Chemical Engineering Science, 143, 226-239, 2016.

9. Deshpande, N. S. \& Barigou, M., Vibrational flow of non-Newtonian fluids, Chemical Engineering Science, 56, 3845-3853, 2001.

10. Haroun, Y., Legendre, D. \& Raynal, L., Volume of fluid method for interfacial reactive mass transfer: Application to stable liquid film, Chem. Eng. Science, 65, 2896-2909, 2010.

11. Ziegenbalg, D., Lob, P., Rawashdeh, M. A., Kralisch, D., Hessel, V. \& Schonfeld, F., Use of 'smart interfaces' to improve the liquid-sided mass transport in a falling film micro reactor, Chem. Eng. Science, 65, 3557-3566, 2010.

12. Woo, B. G. \& Yong, C. K., Melt Polycondensation of Bisphenol A Polycarbonate by Forced Gas Sweeping Process II. Continuous Rotating-Disk Reactor, Ind. Eng. Chem. Res, 40, 3459-3466, 2001.

13. Cai, W., Wang, Y. \& Zhu, S., Flow and mass transfer characteristics in a falling film extractor using hollow fiber as packing, Chem. Eng. J., 108, 161-168, 2005.

14. Schiffer, S., A phenomenological model of dynamic contact angle, Chem. Eng. Science, 55, 59335936, 2000.

15. Fang, C., Hidrovo, C., Wang, F., Eaton, J. \& Goodson, K., 3-D numerical simulation of contact angle hysteresis for microscale two phase flow, International Journal of Multiphase Flow, 34, 690705, 2008.

16. Li, H. Z., Mouline, Y. \& Midoux, N., Modelling the bubble formation dynamics in non-Newtonian fluids, Chem. Eng. Science, 57, 339-346, 2002.

17. Thibault, F. \& Tanguy, P. A., Power-draw analysis of a coaxial mixer with Newtonian and nonNewtonian fluids in the laminar regime, Chem. Eng. Science, 57, 3861 - 3872, 2002.

18. Bird, R. B., Stewart, W. E. \& Lightfoot, E. N., Transport Phenomena, John Wiley \& Sons, Inc., Second Edition, 2007.

19. Kulkarni, S. B., Joshi, S. S. \& Lokhande, C. D., Facile and efficient route for preparation of nanostructured polyaniline thin films: Schematic model for simplest oxidative chemical polymerization, Chem. Engg. J., 166, 1179-1185, 2011. 
20. FLUENT 6.3 User's Guide (2006), Fluent Inc. 
Table 1. Values of the parameters used in the numerical analysis

\begin{tabular}{|c|c|c|c|}
\hline Parameter & Symbol & Value for $\mathrm{X}_{\mathrm{n}}=69$ & Value for $\mathrm{X}_{\mathrm{n}}=82$ \\
\hline Maximum viscosity & $\eta_{0}($ Pa.s $)$ & 47.3 & 86.6 \\
\hline Minimum viscosity & $\eta_{\infty}($ Pa.s $)$ & 2.99 & 5.46 \\
\hline Power law index & $n$ & 0.6 & 0.6 \\
\hline Consistency index & $K\left(\right.$ Pa.s $\left.^{n}\right)$ & 119 & 217.53 \\
\hline Shear rate range & $\dot{\gamma}\left(s^{-1}\right)$ & $10-10000$ & $10-10000$ \\
\hline
\end{tabular}

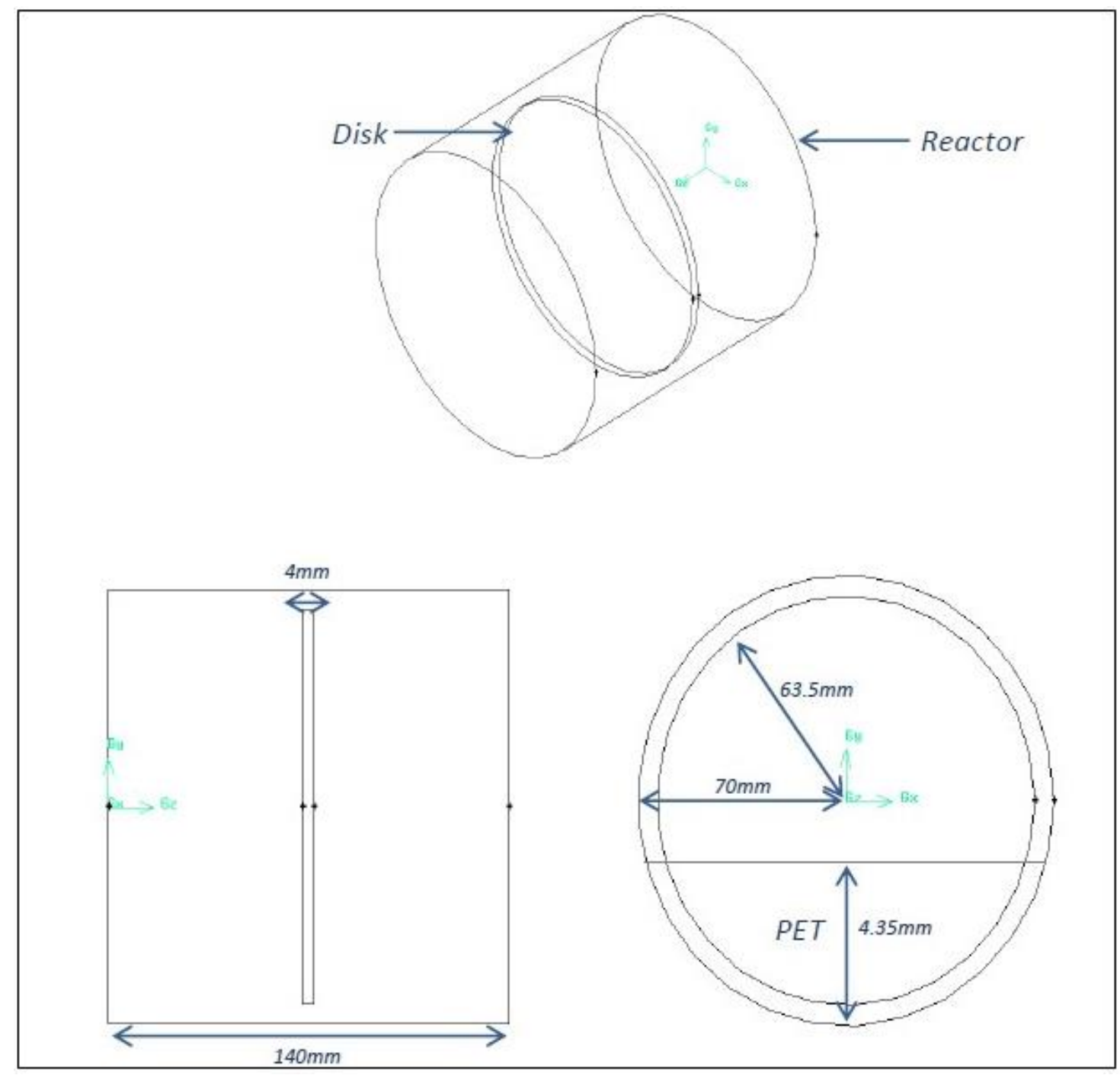

Figure 1 Schematic of the design of the rotating disk reactor 


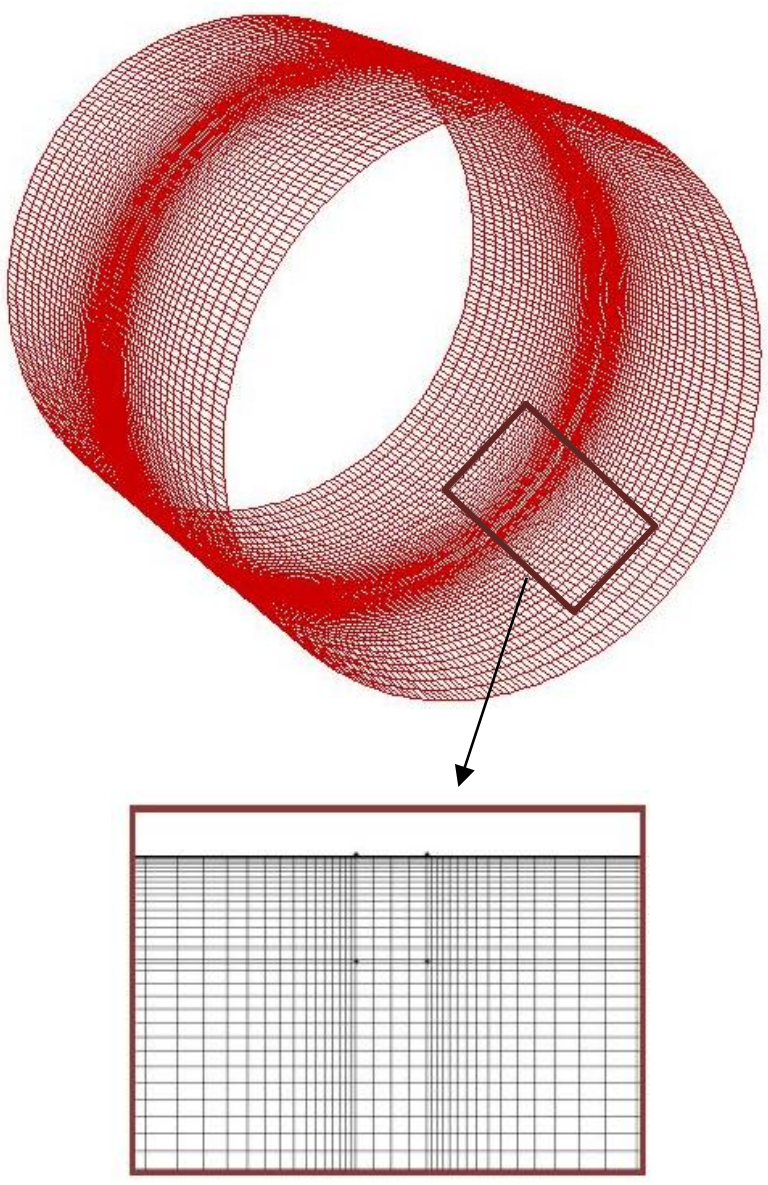

(a) Boundary layer mesh
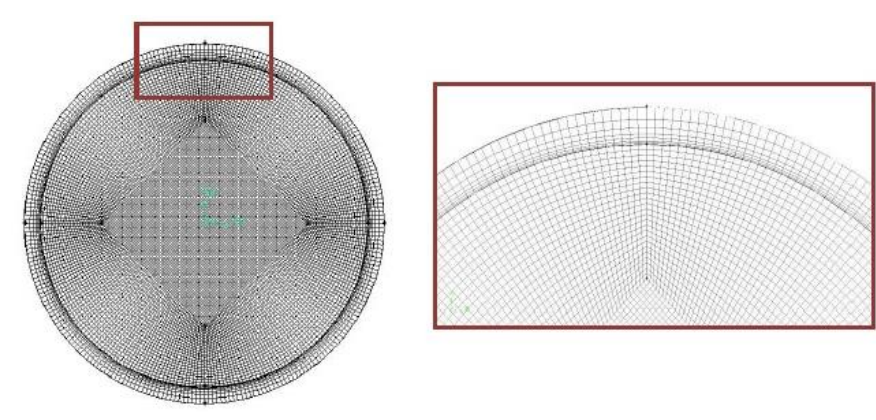

(b) Grid generation on the disk surface

Figure 2. Mesh generation in the reactor 


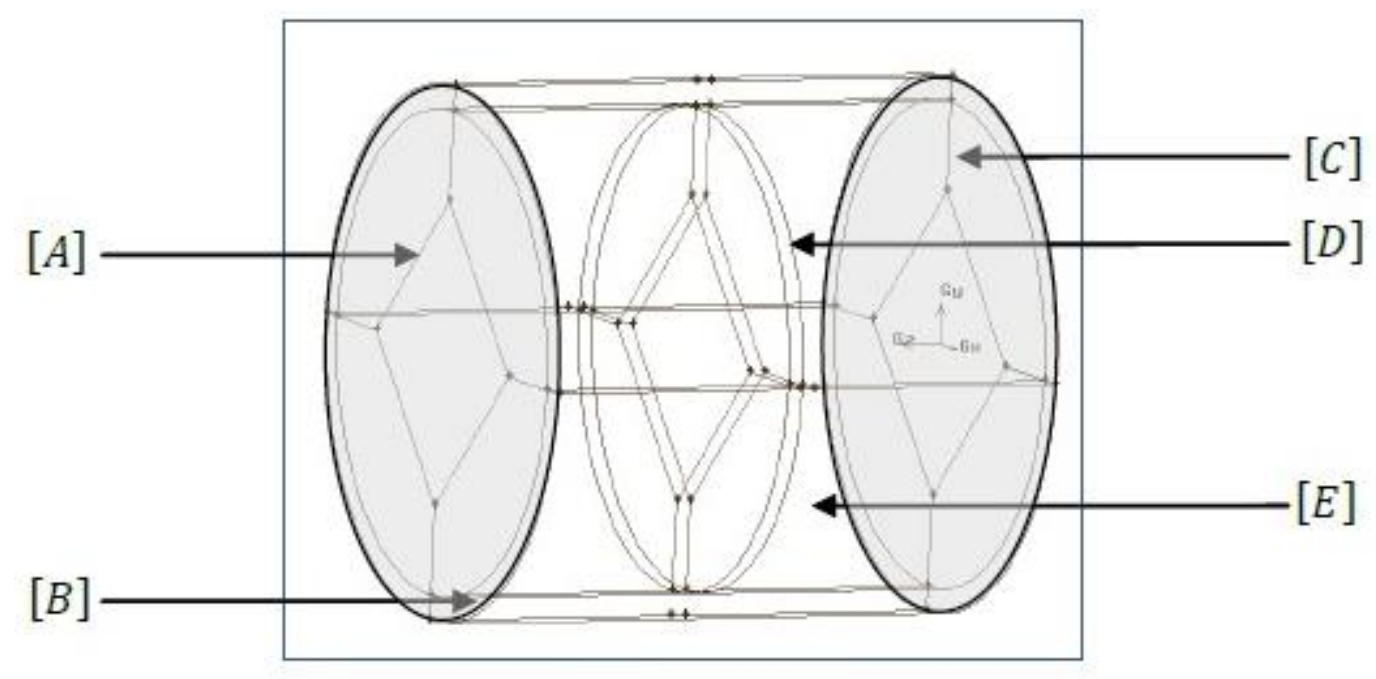

\begin{tabular}{|c|c|c|}
\hline Areas & Description & Boundary condition \\
\hline$[\mathrm{A}]$ & Border of the reactor & Symmetry \\
\hline$[\mathrm{B}]$ & Reactor & Wall \\
\hline$[\mathrm{C}]$ & Border of the reactor & Symmetry \\
\hline$[\mathrm{D}]$ & Disk & Wall \\
\hline$[\mathrm{E}]$ & Fluid area & Interior \\
\hline
\end{tabular}

Figure 3. Boundary conditions

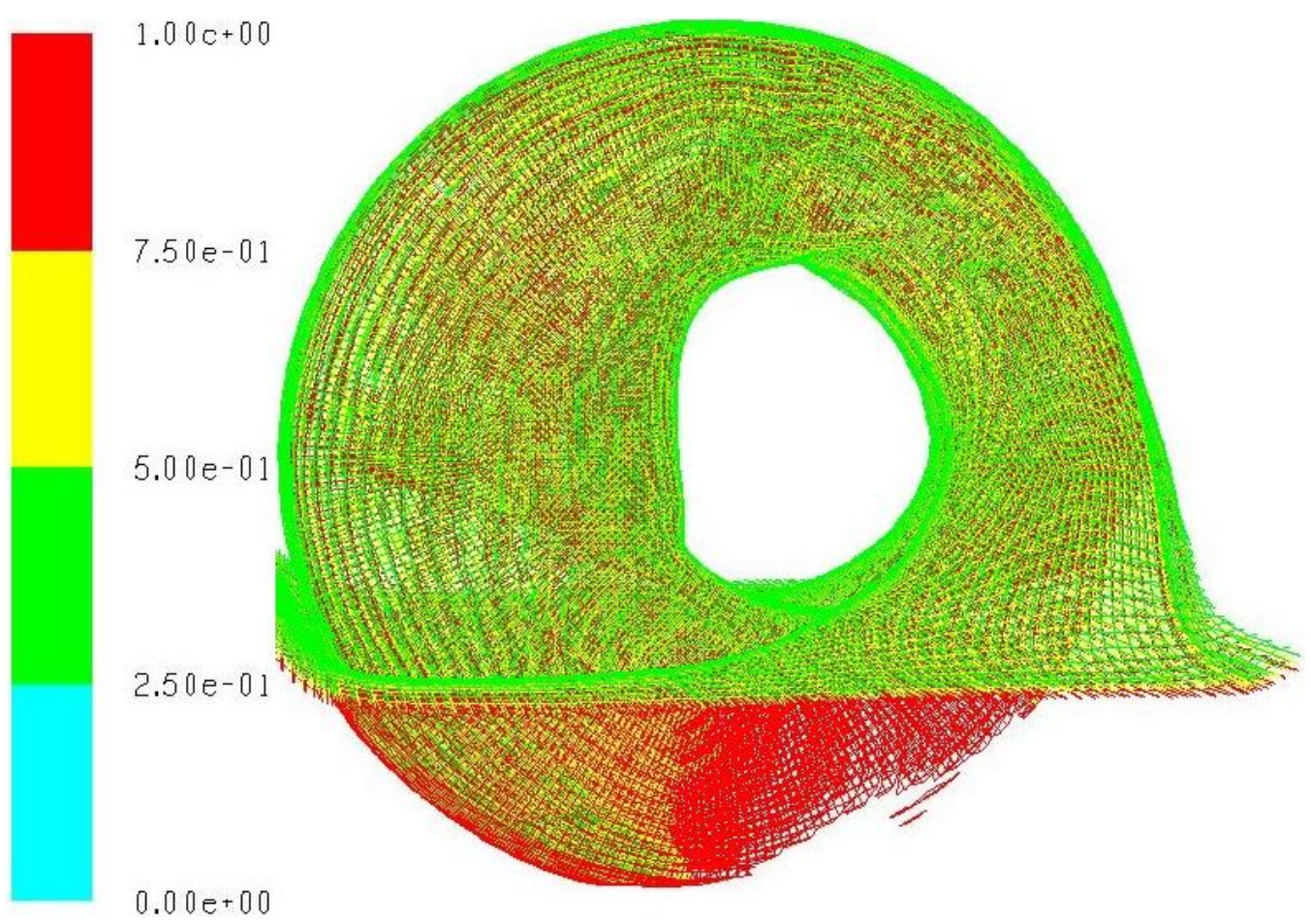

Figure 4. PET film flow pattern $\left(X_{n}=69, \Omega=10 \mathrm{rpm}\right)$ 


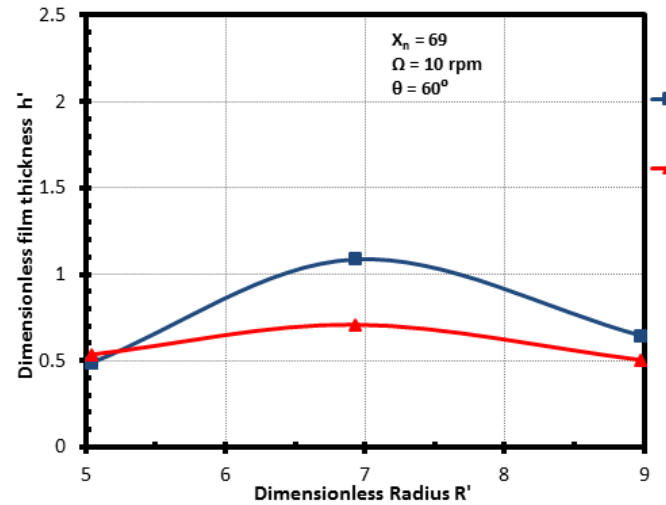

$\theta=60^{\circ}$

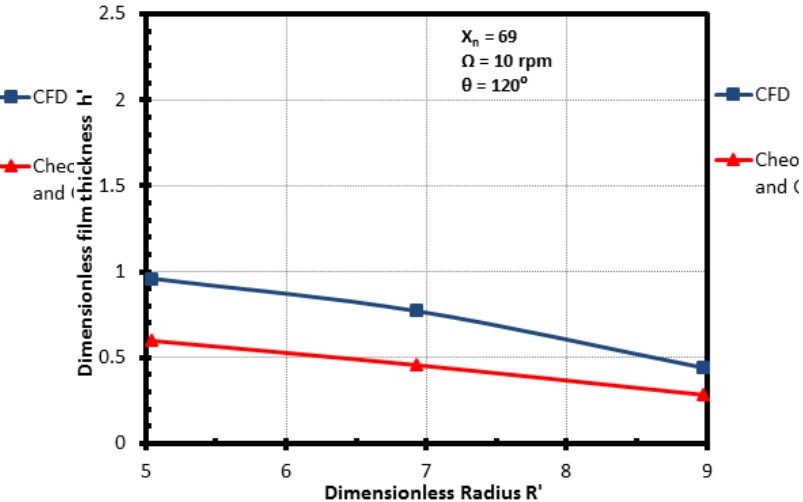

$\theta=120^{\circ}$

Figure 5. Comparison of predicted film thickness with measurement

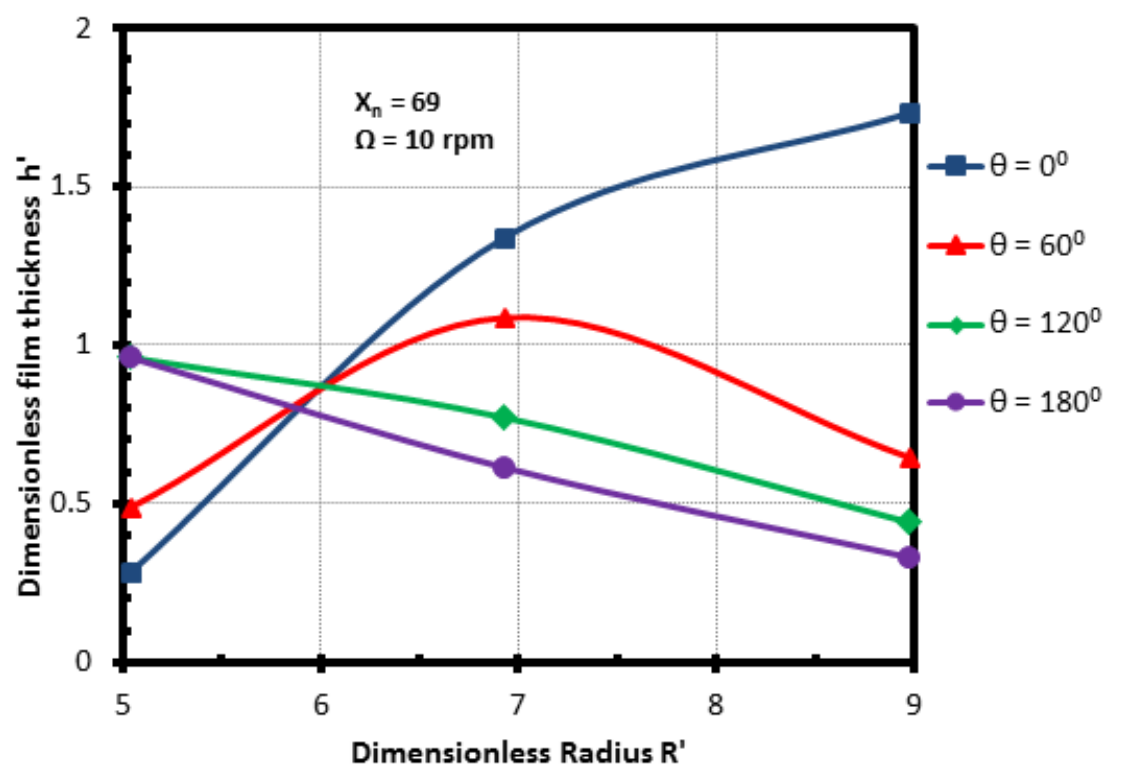

Figure 6. Film thickness for different angular positions 


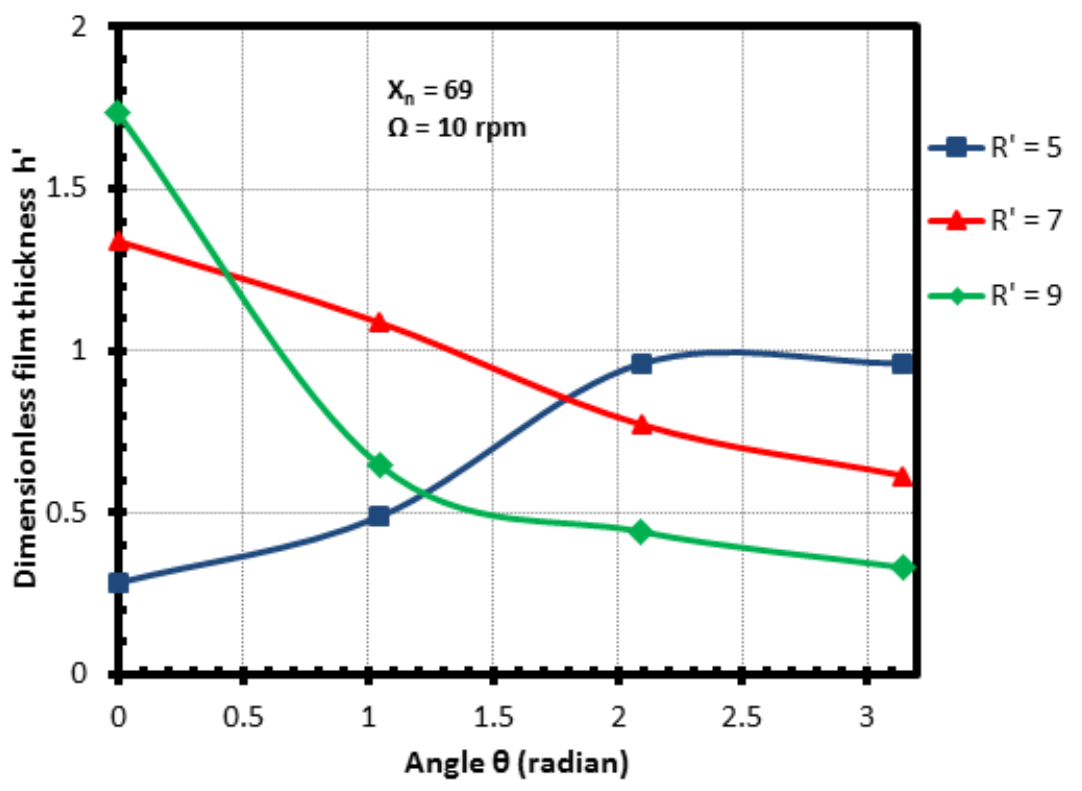

Figure 7. Film thickness for different radial positions

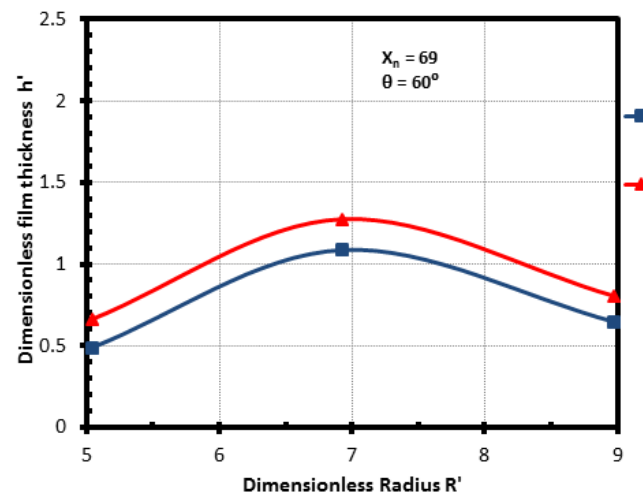

$\theta=60^{\circ}$

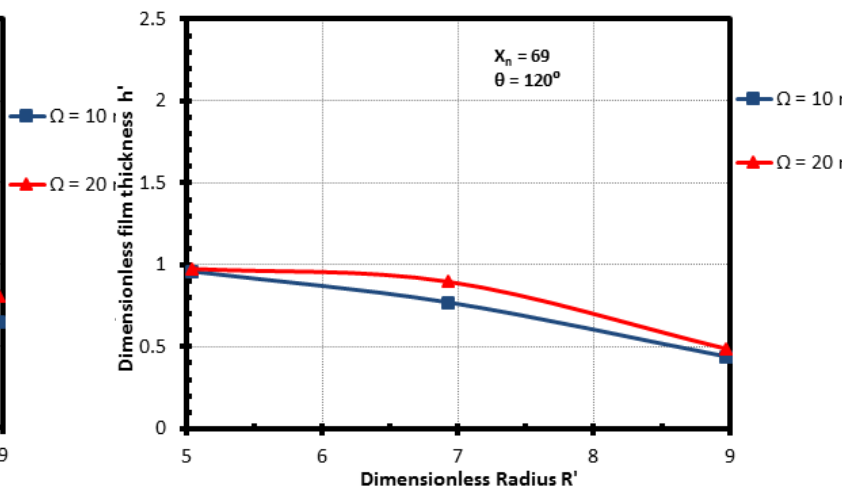

$\theta=120^{\circ}$

Figure 8. Film thickness variation with different rotating speeds

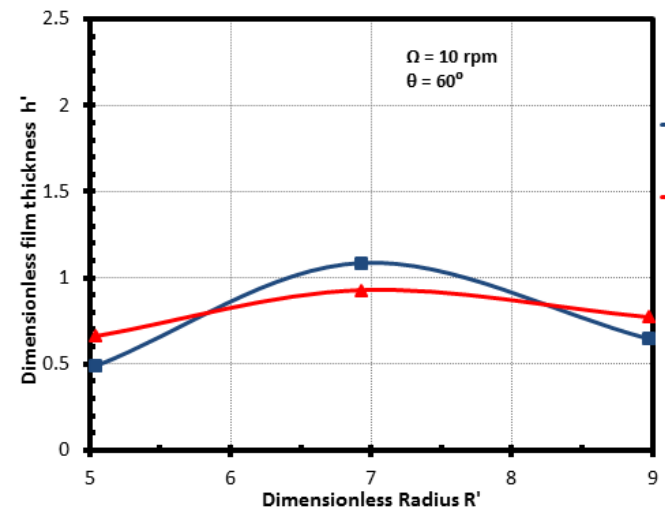

$\theta=60^{\circ}$

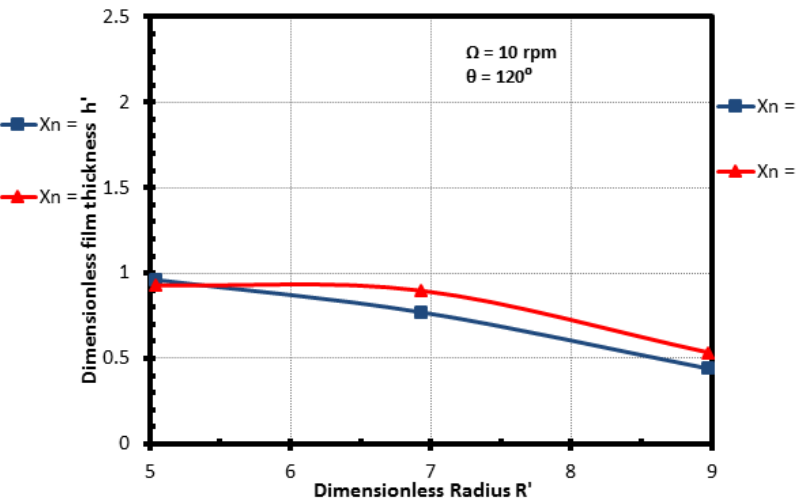

$\theta=120^{\circ}$

Figure 9. Film thickness variation with different molecular weights 


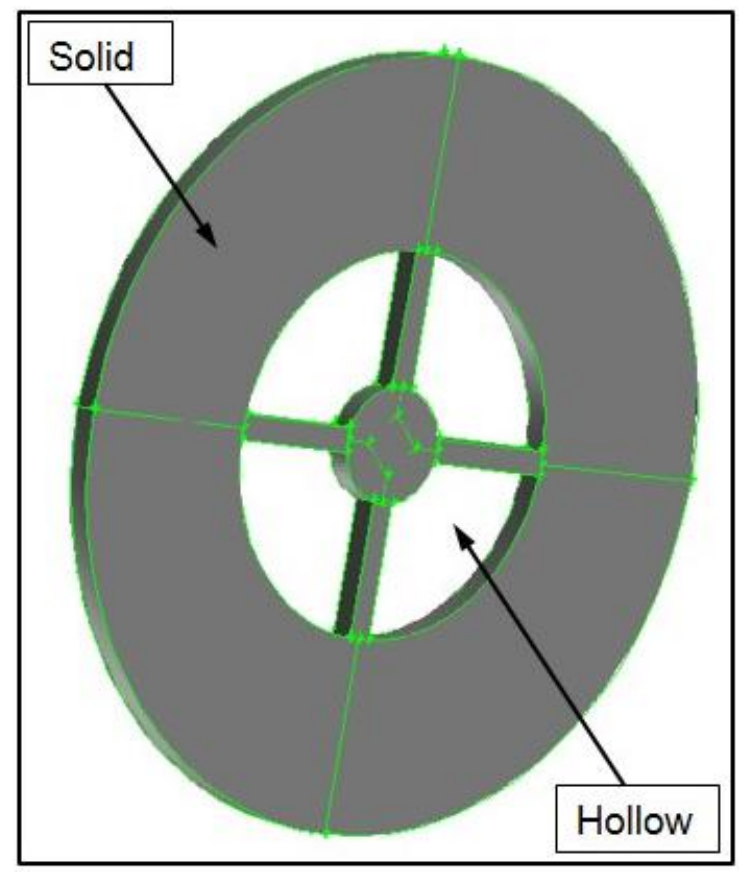

Figure 10. 3D design of the rotating spoked annulus

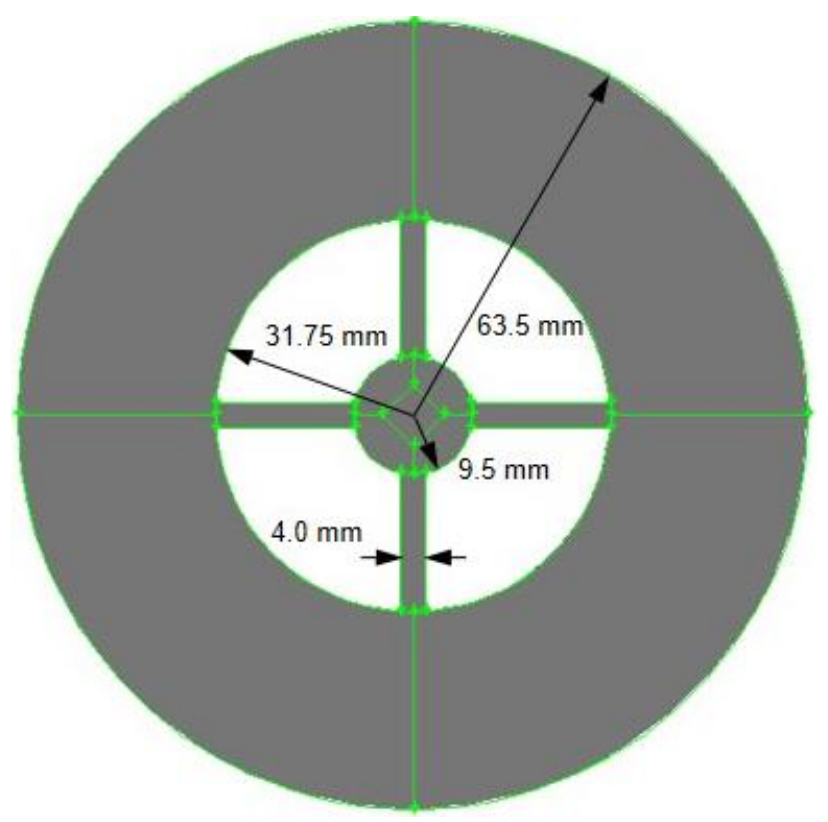

Figure 11. Design specifications of hollow disk 1 


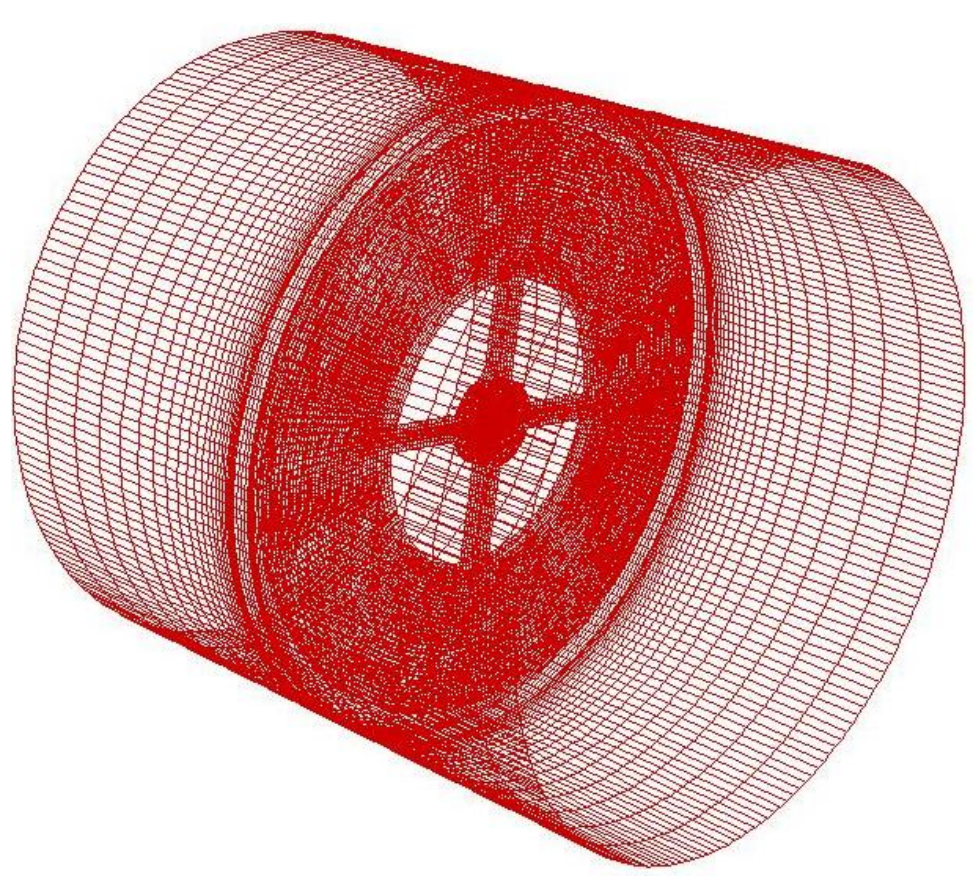

Figure 12. Grid generation on the reactor

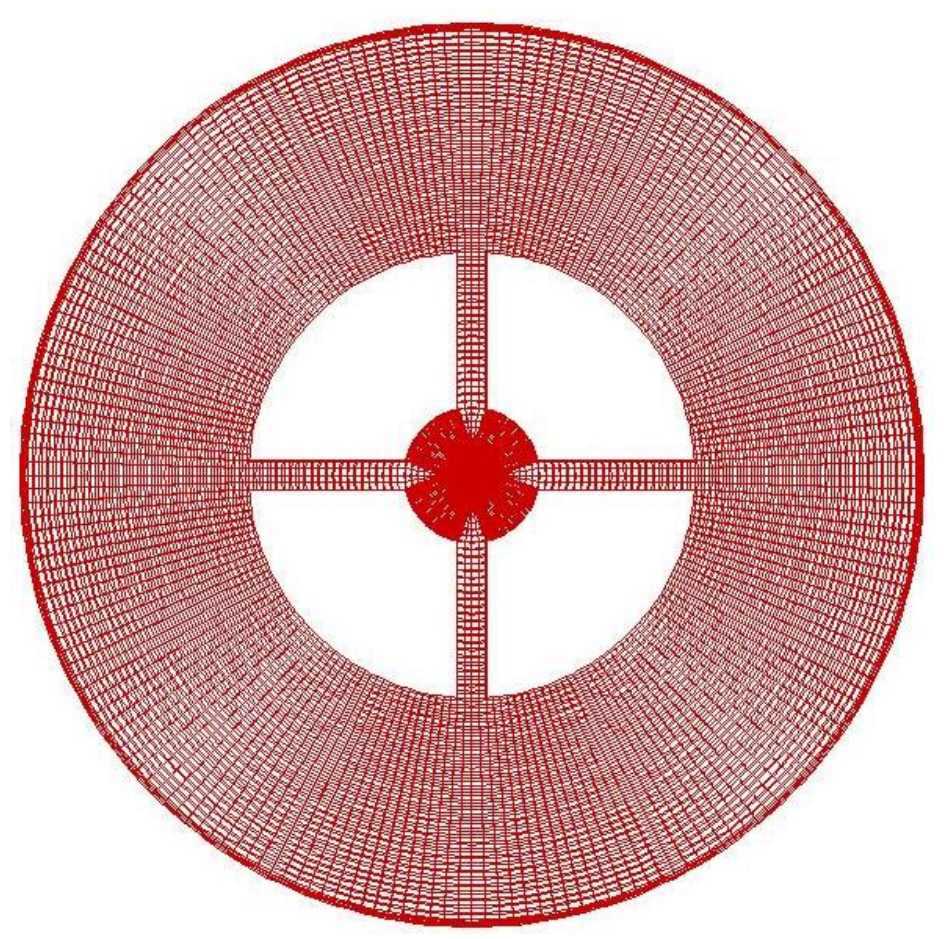

Figure 13. Grid generation on the hollow disk surface 


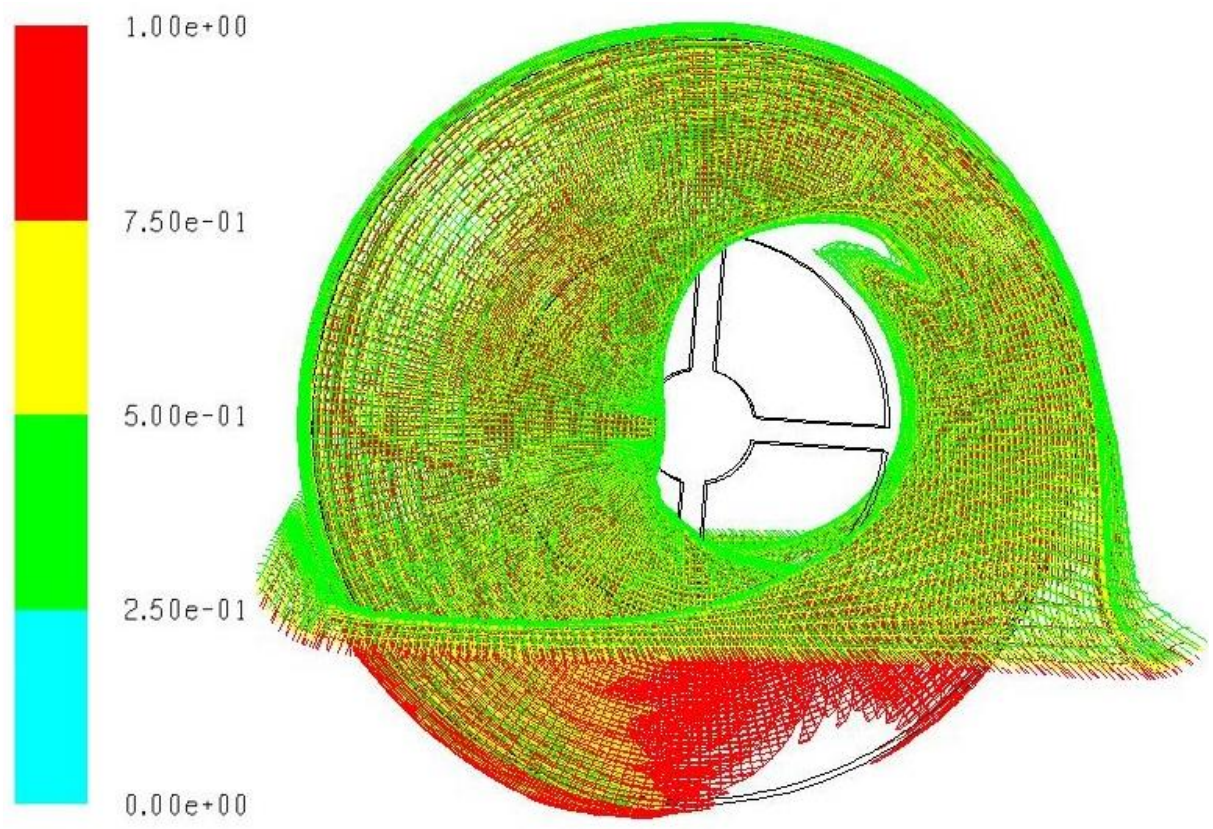

Figure 14. PET film flow pattern on hollow disk1 $\left(X_{n}=69, \Omega=10 \mathrm{rpm}\right)$

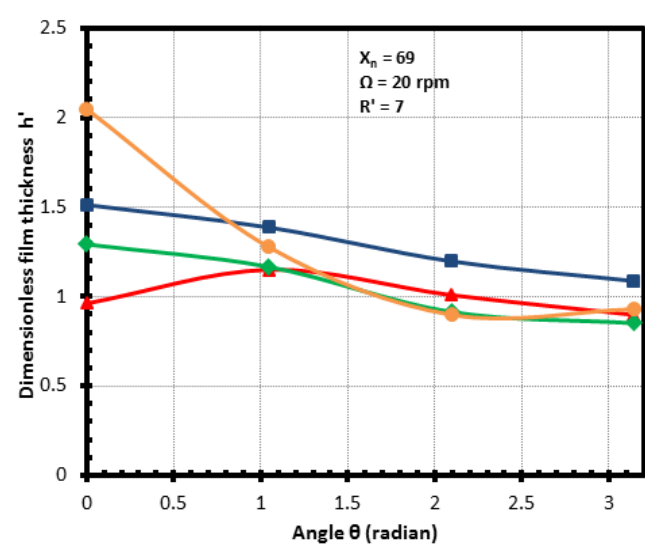

Figure 15a. Comparisons at $\mathrm{R}^{\prime}=7$

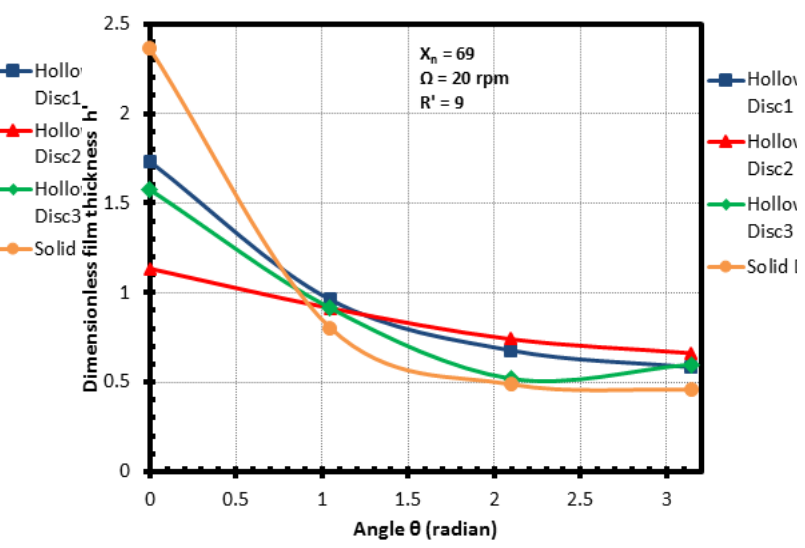

Figure 15b. Comparisons at $\mathrm{R}^{\prime}=9$ 